\title{
An intervention based on the Electronic Medical Record to improve smoking cessation guidance in an urban tertiary care center emergency department
}

\author{
Michael P. Phelan', Balaji Nithianandam², Nathan Eikoff', Daniel Good', Fredric M. Hustey', Stephen Meldon'
}

\begin{abstract}
INTRODUCTION Smoking remains a major public health issue and a leading cause of death and disability in the United States. The objective of this study was to determine the effect of a simple intervention on smoking guidance, based on the electronic medical record (EMR), including providing discharge instructions and/or cessation counseling to emergency department (ED) patients who smoke. METHODS This was an interventional before-and-after study in an ED with 70000 visits per year. A pre-intervention and post-intervention chart review was performed on a random sample of ED visits occurring in 2014 and 2016, identifying smokers and the frequency with which smokers received discharge instructions and/or cessation counseling. In the fall of 2015, our EMR was programmed to deploy smoking cessation discharge instructions automatically. RESULTS In all, 28.7\% (172/600; 95\% CI: 25.2-32.4\%) reported current smoking in the pre-intervention ED population and $27.6 \%$ (166/600; 95\% CI: $24.2-31.4 \%$ ) reported smoking in the post-intervention population. Smoking cessation guidance was provided to a total of $3.5 \%$ of self-reported smokers in the preintervention group $(6 / 172 ; 95 \%$ CI: $1.4-7.6 \%)$; $1.2 \%$ (2/172; 95\% CI: $0.3-$ $4.1 \%)$ were informed of smoking cessation resources as part of their printed ED discharge instructions and $2.3 \%(4 / 172 ; 95 \%$ CI: $0.9-5.8 \%)$ received smoking cessation counseling by the ED provider. There was a statistically significant increase in the proportion of patients receiving any smoking cessation guidance after the intervention. All patients (166/166; 95\% CI: $97-100 \%$ in this period received ED discharge instructions and a list of smoking cessation resources and $3.6 \%$ of smokers $(6 / 166 ; 95 \%$ CI: $1.7-7.7 \%)$ received smoking cessation counseling by the ED provider.

CONCLUSIONS Automated deployment of smoking cessation discharge instructions in the EMR improves smoking cessation discharge instructions, and also has a positive impact on improving rates of in-person counseling by ED providers.
\end{abstract}

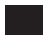 \\ AFFILIATION \\ 1 Cleveland Clinic, Emergency \\ Services Institute, Cleveland, Ohio, \\ United States \\ 2 Metro Health Medical Center, \\ Cleveland, Ohio, United States \\ 3 Northeast Ohio Medical \\ University, Rootstown, Ohio, United \\ States
}

CORRESPONDENCE TO Michael P. Phelan. Cleveland Clinic, Emergency Services Institute, Cleveland, Ohio, United States. E-mail: phelanm@ccf.org

\section{KEYWORDS}

smoking cessation, smoking, emergency medicine, guidance, discharge instructions

\section{Received: 23 August 2018}

Revised: 10 April 2019

Accepted: 11 April 2019

\section{INTRODUCTION}

Tobacco use in the United States remains a significant health problem. Although overall tobacco use has declined, approximately $15 \%$ of adults still report using tobacco. This results in significant health related concerns and costs, particularly among sections of the population such as Medicaid recipients, those below the federal poverty level, and patients with psychiatric issues ${ }^{1-4}$. It will come as no surprise to emergency medicine providers that these groups at significantly higher risk for cigarette smoking parallel the emergency department (ED) patient population. Interventions that have been proven to reduce the prevalence of 
cigarette smoking include raising prices of tobacco products, mass media strategies, removing barriers to access smoking cessation guidance and nicotine replacement products ${ }^{3}$. Many patients who smoke may not seek primary care, leaving the ED as one of the few opportunities to provide smoking cessation counseling to this population. Smoking rates are typically higher among ED patients than in the general population, making an ED visit an opportune time for smoking cessation interventions. There is evidence that a large proportion of ED patients who report a smoking history are willing to initiate steps toward cessation following an ED intervention. There is also good evidence that such ED interventions have a lasting positive effect on smoking cessation success ${ }^{5-7}$. It has also been established that smoking cessation counseling provided by physicians has a modest effect on increasing smoking cessation rates and duration ${ }^{8}$, and given the high public health burden smoking poses, even a small increase in cessation could potentially have a large effect on the population. Unfortunately, patients are rarely given smoking cessation guidance in the form of either printed discharge instructions or smoking cessation counseling during their ED visit. There is evidence that interventions based on an electronic medical record may provide an advantage with regard to smoking cessation support provided by physicians in the ambulatory environment ${ }^{9}$. The effect of EMR-facilitated interventions on smoking cessation actions has been reviewed by the Cochran group that evaluated the available evidence supporting computerized medical record systems as a way to enhance the delivery of effective tobacco use guidance and treatment. The finding suggests that there is a potential for the EMR to facilitate some evidence-based tobacco interventions ${ }^{10}$. This type of benefit may translate to the ED as well.

\section{Objective}

The objective of this study was to determine the effect of interventions based on an electronic medical record (EMR) in providing patients with any smoking cessation guidance, focusing on discharge instructions on quitting for all identified ED patients who smoke and evaluating if any smoking cessation counseling was provided.

\section{METHODS}

This was an interventional before-and-after study conducted in an urban tertiary care ED with an affiliated residency program and approximately 70000 patient visits per year. As this study was deemed minimal risk, it was granted exemption by the institutional review board. A retrospective chart review was performed on a consecutive sample of 600 ED visits occurring in 2014 before the intervention and again post-intervention in 2016, to identify rates of smoking cessation counseling and resource information in discharge instructions provision. Charts were reviewed by trained reviewers using a standardized data abstraction tool. A patient was identified as a smoker if current tobacco smoking was noted in the social history component of the EMR. It was standard practice in the study site ED to obtain a social history on every patient, at minimum including whether the patient had a current history of cigarette smoking. Smoking cessation guidance was defined as documentation in the EMR of counseling by the ED physician and/or discharge instructions specifically related to smoking cessation. Any language documented by physicians or advanced practice providers in the medical record regarding discussion of the hazards of smoking or regarding recommendations to quit smoking was accepted as evidence of smoking cessation counseling. During the pre-intervention period (usual care), smoking cessation guidance was left to the discretion of the care providers without prompting. In the fall of 2015, our EMR was programmed to automatically deploy smoking-specific discharge instructions if current tobacco use was identified. These instructions included information on the dangers of smoking, methods of quitting, as well as the CDC's QUITNOW call line. An EMR smart phrase was also developed (".edsmoking") as an option to make it easier to document smoking cessation counseling, and providers were educated about its use. It is standard practice at the study site ED for nurses to review all discharge instructions with the patient at the time of discharge, including discharge instructions on smoking cessation guidance. Smoking cessation guidance rates were compared between the two groups. Proportions with 95\% confidence intervals (CI) using the modified Wald method are reported for comparisons across groups. 


\section{RESULTS}

Of the 600 patients included in the pre-intervention arm, 28.7\% (172/600; 95\% CI: 25.9-32.4\%) reported current smoking. Any smoking cessation guidance was given to a total of $3.5 \%$ of selfreported smokers $(6 / 172 ; 95 \%$ CI: $1.4-7.6 \%)$; $2.3 \%$ (4/172; $95 \%$ CI: $0.9-5.8 \%)$ received smoking cessation counseling by the ED physician/APP and $1.2 \%(2 / 172 ; 95 \%$ CI: $0.3-4.1 \%)$ were informed of smoking cessation resources as part of their printed ED discharge instructions. Of the 600 patients in the post-intervention arm, 27.6\% (166/600; 95\% CI:
$24.2-31.4 \%)$ reported current smoking. There was a statistically significant increase in the proportion of patients receiving smoking cessation guidance after the intervention. All patients (166/166; 95\% CI: $97-100 \%$ ) in this period received ED discharge instructions and a list of smoking cessation resources and $3.6 \%$ of smokers (6/166; 95\% CI: $1.7-7.7 \%)$ received smoking cessation counseling by the ED provider (Figure 1). There was a small increase in documentation of physician smoking cessation counseling after the intervention but this was not statistically significant.

Figure 1. Comparison of smokers who received discharge instructions and smoking cessation guidance in 2014 and 2016 after EMR fix was deployed to provide smokers identified in the social history with discharge instructions

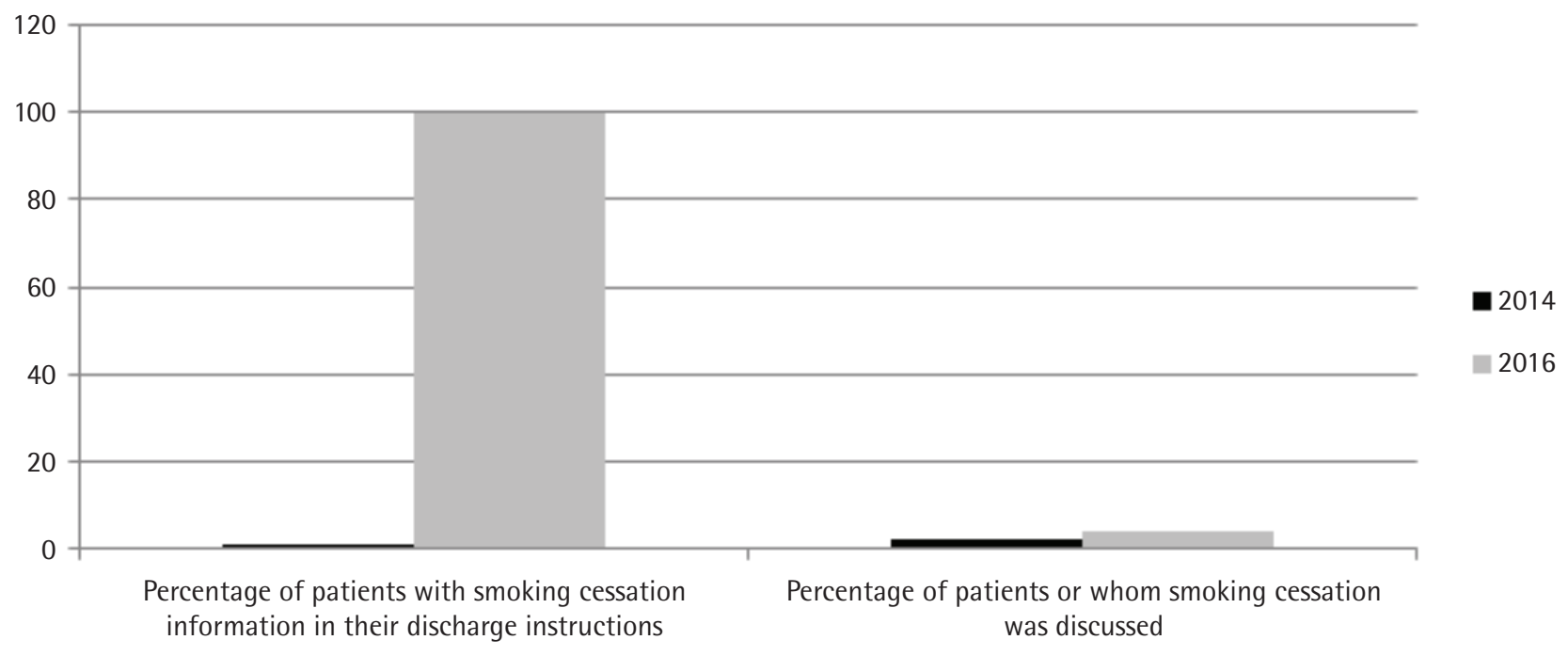

\section{DISCUSSION}

Our data indicate that smoking remains a major problem among ED patients. Given that patients who are evaluated in an ED setting do not often have primary care providers for follow-up care, it is worthwhile to provide counseling for them. According to Medicaid and the Affordable Care Act (ACA), this is a billable activity so long as the physician discusses smoking cessation with the patient for at least 3 minutes, which occurred twice in our study group. Obviously, physician education is needed to heighten awareness of the opportunity presented when a current smoker is being treated in the ED.

The EMR can be used as an effective tool for identifying smokers and thus alerting the physician to the opportunity to provide smoking cessation instructions. We found this approach to be relatively easy to implement and anticipate that it can be duplicated at other medical care facilities. Patients could also be assisted in finding a primary care provider to follow up on their attempts to stop smoking. Other automated interventions embedded into the EMR such as a best practice alert to remind caregivers of the opportunity for counseling might work synergistically with our EMR intervention to enhance its effect.

\section{Limitations}

Our study has several limitations. It was based on a retrospective chart review. It is possible that smoking 
cessation guidance was provided but not documented in either phase of the study, affecting our overall results. The analysis also did not take into account the possibility of clustering by the emergency clinician. However, the large number of providers working in the ED during the study period with the opportunity to provide counseling is likely to have mitigated this effect. In addition, while evidence exists that any smoking cessation provided by physicians increases the rate of actual smoking cessation ${ }^{8}$, our study was not designed to evaluate the effect of an EMR-based intervention on actual smoking cessation rates.

\section{CONCLUSIONS}

Smoking is a major cause of morbidity and mortality, and a large portion of ED patients are active smokers. The EMR can be used to generate written discharge instructions that include strategies and resources for support with smoking cessation, providing all selfreported smokers with valuable resources. Such an intervention also appears to modestly increase the rates of in-person smoking cessation counseling provided by ED physicians and advanced practice providers. While the effect may be modest, even small increases in smoking cessation counseling can have significant public health implications.

\section{REFERENCES}

1. Centers for Disease Control and Prevention (CDC). Smoking-attributable mortality, years of potential life lost, and productivity losses--United States, 2000-2004. MMWR Morb Mortal Wkly Rep. 2008;57(45):1226-1228.

2. U.S. Department of Health and Human Services. The Health Consequences of Smoking-50 Years of Progress: A Report of the Surgeon General. Atlanta: U.S. Department of Health and Human Services, Centers for Disease Control and Prevention, National Center for Chronic Disease Prevention and Health Promotion, Office on Smoking and Health; 2014.

3. Jamal A, King BA, Neff LJ, Whitmill J, Babb SD, Graffunder CM. Current cigarette smoking among adults - United States, 2005-2015. MMWR Morb Mortal Wkly Rep. 2016;65(44):1205-1211. doi:10.15585/mmwr.mm6544a2

4. Centers for Disease Control and Prevention (CDC). Cigarette smoking among adults and trends in smoking cessation - United States, 2008. MMWR Morb Mortal Wkly Rep. 2009;58(44):1227-1232.

5. Boudreaux ED, Baumann BM, Friedman K, Ziedonis DM. Smoking stage of change and interest in an emergency department-based intervention. Acad Emerg Med. 2005;12(3):211-218. doi:10.1111/j.1553-2712.2005.tb00871.x

6. Bernstein SL, D’Onofrio G, Rosner J, et al. Successful tobacco dependence treatment in low-income emergency department patients: a randomized trial. Ann Emerg Med. 2015;66(2):140-147. doi:10.1016/j.annemergmed.2015.03.030

7. Berstein SL, Boudreaux ED, Cydulka RK, et al. Tobacco control intervention in the emergency department: a joint statement of emergency medicine organizations. Ann Emerg Med. 2006;48(4):e417-e426. doi:10.1016/j.annemergmed.2006.02.018

8. Stead LF, Buitrago D, Preciado N, Sanchez G, HartmannBoyce J, Lancaster T. Physician advice for smoking cessation. Cochrane Database of Systematic Reviews. 2013;5. doi:10.1002/14651858.cd000165.pub4

9. Bae J, Ford EW, Huerta TR. The Electronic Medical Record's Role in Support of Smoking Cessation Activities. Nicotine Tob Res. 2016;18(5):1019-24. doi:10.1093/ntr/ntv270

10. Boyle R, Solberg L, Fiore M. Use of Electronic Health Records to Support Smoking Cessation. Cochrane Database of Systemic Reviews. 2014;12. doi:10.1002/14651858.cd008743.pub3

ACKNOWLEDGEMENTS

The manuscript was copyedited by L.J. Kesselring.

\section{CONFLICTS OF INTEREST}

The authors have completed and submitted the ICMJE Form for Disclosure of Potential Conflicts of Interest and none was reported.

\section{FUNDING}

There was no source of funding for this research.

PROVENANCE AND PEER REVIEW

Not commissioned; externally peer reviewed. 